\title{
Attribution of horizontal and vertical contributions to spurious mixing in an Arbitrary Lagrangian-Eulerian ocean model
}

\author{
Angus H. Gibson ${ }^{\mathrm{a}}$, Andrew McC. Hogg ${ }^{\mathrm{a}}$, Andrew E. Kiss ${ }^{\mathrm{b}}$, \\ Callum J. Shakespeare ${ }^{\mathrm{a}}$, Alistair Adcroft ${ }^{\mathrm{c}}$ \\ ${ }^{a}$ Research School of Earth Sciences and ARC Centre of Excellence for Climate System \\ Science, Australian National University, Canberra, Australian Capital Territory, Australia \\ ${ }^{b}$ School of Physical, Environmental and Mathematical Sciences, University of New South \\ Wales at the Australian Defence Force Academy, Canberra, Australian Capital Territory, \\ Australia \\ ${ }^{c}$ NOAA Geophysical Fluid Dynamics Laboratory, Princeton, New Jersey, USA
}

\begin{abstract}
We examine the separate contributions to spurious mixing from horizontal and vertical processes in an ALE ocean model, MOM6, using reference potential energy (RPE). The RPE is a global diagnostic which changes only due to mixing between density classes. We extend this diagnostic to a sub-timestep timescale in order to individually separate contributions to spurious mixing through horizontal (tracer advection) and vertical (regridding/remapping) processes within the model. We both evaluate the overall spurious mixing in MOM6 against previously published output from other models (MOM5, MITGCM and MPAS-O), and investigate impacts on the components of spurious mixing in MOM6 across a suite of test cases: a lock exchange, internal wave propagation, and a baroclinically-unstable eddying channel.

The split RPE diagnostic demonstrates that the spurious mixing in a lock exchange test case is dominated by horizontal tracer advection, due to the spatial variability in the velocity field. In contrast, the vertical component of spurious mixing dominates in an internal waves test case. MOM6 performs well in this test case owing to its quasi-Lagrangian implementation of ALE. Finally, the
\end{abstract}

Email address: angus.gibson@anu.edu.au (Angus H. Gibson)

Preprint submitted to Ocean Modelling

September 15, 2017 
effects of model resolution are examined in a baroclinic eddies test case. In particular, the vertical component of spurious mixing dominates as horizontal resolution increases, an important consideration as global models evolve towards higher horizontal resolutions.

\section{Introduction}

One of the myriad uses of ocean models is in developing ocean heat uptake estimates and overturning circulation predictions (Armour et al. 2016). Additionally, the overturning circulation itself affects the wider climate, which 5 manifests when ocean models are used as a component of coupled climate simulations. The strength of ocean heat uptake and the overturning circulation are both strongly controlled by the density structure of the ocean, which is modified by mixing. For example, mixing at depth modifies the abyssal overturning cell that constitutes part of the meridional overturning circulation (Mashayek et al. 2015), while the time scale of adjustment of the overturning circulation toward equilibrium is sensitive to near-surface mixing (Vreugdenhil et al. 2015). A consequence of this sensitivity is that ocean models with significant mixing due to numerical truncation errors (spurious mixing) are unlikely to accurately constrain the abyssal overturning.

Numerical ocean models are governed by approximations of the incompressible Navier-Stokes equations for momentum, also known as the primitive equations (Griffies 2004). In these models, the vertical balance is hydrostatic, where the vertical pressure gradient force is matched by the gravitational force. The mixing of momentum by the unresolved eddy field from the mesoscale down to the Kolmogorov scale is parameterised by an explicit eddy viscosity term. Potential density of water parcels is a function of salinity and potential temperature through an equation of state. These tracers are advected by the explicitly resolved eddy field, and mixed by the unresolved eddy field through a parameterised eddy diffusivity term. Due to the vast difference between lateral and vertical scales in models, the eddy viscosity and diffusivity terms are often sep- 
arated into lateral and vertical components.

To solve the primitive equations, ocean models implement some kind of discretisation, such as the finite volume method. This discretisation involves representing the computational domain as a series of grid cells in three-dimensional

30 space, where each grid cell has associated mean velocities and tracer concentrations, and possibly higher moments (Prather 1986). Horizontal tracer advection schemes are discretisations of the advection equation that use information from neighbouring grid cells to create higher-order reconstructions of the tracer field than that which is stored directly in the cell. Mixing processes create fluxes of tracer between grid cells. In ocean models, mixing has two main causes, physical and numerical. The physical mixing comes from advection by numerically unresolved turbulence, which is typically parameterised as a diffusive process. On the other hand, numerical mixing arises from truncation errors in the discretisations and algorithms used by the ocean model to solve the governing equations.

40 Numerical mixing is also known as spurious mixing and has no physical basis. For example, first-order upwind advection has numerical diffusion as the leading error term (Gentry et al. 1966).

Spurious mixing is undesirable in ocean models as it is unphysical and may add to the imposed and parameterised mixing to an unknown extent. Spuri45 ous mixing affects numerical experiments which are contingent on the density structure of the ocean. Ocean heat uptake or overturning circulation strength in such experiments may be biased (Griffies et al. 2015). One of the considerations in model development and configuration is thus to ensure spurious mixing is minimised.

The magnitude of spurious mixing is strongly influenced by the choice of horizontal tracer advection scheme. Much of the focus in reducing spurious mixing has therefore been on tracer advection, through improving numerical accuracy or the model's tracer sub-gridscale representations. Some argue that a high-order advection scheme is sufficient to reduce the spurious mixing to ${ }_{55}$ acceptable levels (Daru and Tenaud 2004). This is simply a matter of using a sufficiently high-order polynomial reconstruction to try to capture the overall 
structure of tracer distributions. Other advection schemes attempt to preserve the sub-gridscale representation of a given field. For example, by carrying information about both first and second-order moments, the Prather (1986) method

${ }_{60}$ is able to reconstruct a field to second order. This second-order moment scheme must often be used in conjunction with a flux limiter to avoid the creation of spurious minima and maxima; these limiters in effect lead to a sub-cell diffusion (Morales Maqueda and Holloway 2006). An alternative view is that the tracer advection scheme only needs sufficient accuracy before grid-scale noise in velocity becomes the dominant source of spurious mixing (Illcak et al. 2012).

A second consideration in model configuration in order to minimise spurious mixing is the vertical coordinate. We first describe some of the main choices for the vertical coordinate in ocean models. The basis for the z-family of coordinates is the pure z-level coordinate, where coordinate surfaces are simply fixed 70 geopotentials. The first extension to the z-level coordinate is $z^{*}$ or z-star, which individually and uniformly expands or contracts water columns to accomodate changes in the free surface height (Adcroft and Campin 2004). z-family coordinates allow for ahead-of-time specification of vertical resolution, which must be applicable to the entire modelled domain and thus sufficiently general. Some disadvantages of these coordinates are poor representation of overflows (?), and the spurious diapycnal mixing associated with purely horizontal coordinate surfaces, e.g. isoneutral diffusion (Griffies et al. 2000). Instead of being referenced to physical positions, isopycnal coordinate models use potential density as the vertical coordinate. This formulation completely eliminates spurious diapycnal mixing, as well as providing enhanced vertical resolution at sharp density fronts. However, there are difficulties in representing the nonlinear equation of state, as there is no conservative density coordinate that is monotonic with depth (Griffies 2004). Additionally, the surface mixed layer is essentially unstratified, and hence is a region of very low vertical resolution.

Hybrid vertical coordinates combine or modify other vertical coordinates to optimise their performance, at the expense of complexity and computational cost. One hybrid vertical coordinate is z-tilde (Leclair and Madec 2011), which 
has Lagrangian behaviour (i.e., the grid is advected by the vertical velocity) for motions on short timescales, but relaxes to a target z-star grid over long timescales to prevent the grid from drifting. This scheme was demonstrated to reduce spurious mixing when modelling the propagation of internal gravity waves. A final example is the continuous isopycnal coordinate (White et al. 2009), where instead of layers having a predefined density as in the pure isopycnal coordinate, interfaces have a target density. In this case, there must be dynamic adjustment of the coordinate surfaces in order to maintain the target density. The release of the constraint to layered isopycnals means that further physical processes can be more easily added to the model, such as geothermal heating or double diffusion (White et al. 2009). In isolation, each coordinate has strengths and weaknesses for ocean modelling, but the combination attempts to preserve the strengths of each.

To allow generalised vertical coordinates, models can make use of an arbitrary Lagrangian-Eulerian (ALE) scheme. There are two general implementations of ALE in ocean models, depending on the reference frame of the model (Margolin and Shashkov 2003, Leclair and Madec 2011). In quasi-Eulerian models, any changes in the vertical grid due to the choice of coordinate are incorporated into the solution of the primitive equations (Kasahara 1974). Incorporating changes in the vertical grid is often done by calculating the motion of the new vertical grid relative to the old grid as a vertical velocity. As such, there could be an associated spurious mixing with advection in both the horizontal and vertical directions.

The quasi-Lagrangian algorithm (Hirt et al. 1974, Bleck 2002) is for models which are primarily implemented in a Lagrangian frame of reference (such as MOM6, which is the focus of this paper; see Jansen et al. 2015). Here, the vertical grid may adjust during the dynamic solution of the primitive equations or as a consequence of parameterisations such as Gent-McWilliams thickness diffusion (Gent and McWilliams 1990). This dynamical timestep incorporates most of the modelled processes, including the calculation and application of advective tracer fluxes. Typically, these fluxes are accumulated during the so- 
lution of the primitive equations and applied after the primitive equations are solved, on the updated grid. The model dynamics are then followed by the ALE timestep, which consists of two phases. In the first phase, referred to here as regridding, a new vertical grid is calculated using the current model state. This new grid may be as simple as a prescribed z-star coordinate, or could be a function of local density or depth. Secondly, the new grid is applied in the remapping phase, during which the model state is mapped onto the new grid. The remapping algorithm is often an adaptation of an advection scheme (Margolin and Shashkov 2003), although other conservative algorithms may be used. Remapping differs from vertical advection in that the effective vertical velocities have a non-physical component to recover the new grid, as well as a physical component. Spurious mixing that occurs during the remapping phase therefore depends on the vertical dynamics, the new grid and the sub-gridscale reconstruction of tracers on the old grid.

The accuracy of the reconstruction scheme used in the remapping stage of ALE was investigated by White and Adcroft (2008) with their piecewise quartic method (PQM). PQM is the most accurate reconstruction method available in MOM6, and was found to significantly increase reconstruction accuracy for a small increase in computational cost compared to limited PPM (piecewise parabolic method). The impacts of different reconstruction schemes in regridding and remapping were considered by White et al. (2009), comparing their ${ }_{140}$ spurious mixing in terms of the change of volume distributions across density classes. Neither of these studies quantified the magnitude of spurious mixing in total, or as a comparison to the spurious mixing by horizontal advection. Formulating this comparison is one of the aims of this paper.

There is no consensus on the appropriate diagnostic technique to use to evaluate the performance of numerical schemes with regard to spurious mixing. Griffies et al. (2000) used an effective diapycnal diffusivity, which allows for direct comparison between the spurious mixing and expected oceanic values. However, because it uses a reference density profile compiled from the entire domain, the effective diffusivity is only a single idealised vertical profile, and 
cannot be mapped back to real space.

An alternative to diagnosing spurious mixing from the model state is to calculate an analytical solution from the advection operator itself. Morales Maqueda and Holloway (2006) used this approach for upstream based schemes, such as the second-order moment method of Prather (1986), calculating a closed form expression for the implicit numerical diffusivity.

Substituting the second-order moment scheme for an arbitrary choice of horizontal advection scheme, Burchard and Rennau (2008) inferred the effect of sub-gridscale structure on the destruction of the spatial variance of a tracer, which was adapted to a finite volume framework by Klingbeil et al. (2014). A tracer variance diagnostic thus compares physical and numerical mixing through sub-gridscale changes. Tracer variance can be calculated for every model gridpoint, and its destruction gives information about the relative impact of physical and numerical mixing through full space.

An alternative diagnostic of spurious mixing is to measure its effect on the reference potential energy RPE (Winters et al. 1995) when all explicit diapycnal terms are set to zero. This metric gives only timeseries data (no localised information), but allows for ready comparison across models with the same physical configuration. The comparison across models allows for a quantitative evaluation of algorithms or parameters that differ across models, or within the same model. The global RPE diagnostic can be extended to provide a localised density of reference potential energy (Ilicak 2016), alleviating one of the shortcomings of the global quantity, and showing, for example, that regions of high shear are the most susceptible to spurious mixing.

Ilicak et al. (2012) used the rate of change of RPE in analysing the role of momentum closure in different models (GOLD, MITGCM, MOM5 and ROMS). Comparisons were performed across a suite of test cases intended to stress different physical phenomena: a lock exchange, downslope flow, internal gravity waves, baroclinic eddies, and a global spindown. Ilicak et al. (2012) studied the 
dependence of spurious mixing on the lateral grid Reynolds number

$$
\operatorname{Re}_{\Delta}=\frac{U \Delta x}{\nu_{h}},
$$

This paper has two main aims. Firstly, to evaluate the performance of an- 
other ALE model, MOM6, against the models studied by Ilicak et al. (2012) and Petersen et al. (2015). The comparison is made using both the z-star and z-tilde coordinates, as well as the continuous isopycnal (rho) coordinate that is unique to MOM6. Secondly, a method is proposed for using RPE changes to separate the contributions of horizontal and vertical processes (i.e. advection and ALE) to the spurious mixing. This method allows for the evaluation of different advection schemes, and different orders of reconstruction in ALE, and is proposed as a new tool to compare the performance of different vertical coordinates.

\section{Theory}

\subsection{Reference potential energy}

A measurement of spurious mixing with a physical basis comes from the reference potential energy (RPE, known also as background potential energy; Winters et al. 1995). The RPE is the lowest potential energy attainable by rearrangement of a given fluid so that there is no energy available for motion. To calculate this state, the fluid must be adiabatically re-sorted to a stable onedimensional stratification. The sum of the reference potential energy and the ${ }_{225}$ available potential energy (APE) gives the total potential energy in the system. Mathematically, the RPE is expressed as

$$
\mathrm{RPE}=g \int_{\Omega} z \rho^{*}(z) \mathrm{d} V,
$$

where $g$ is the gravitational constant; $z$ is the height, positive upward; $\Omega$ is the domain; $V$ is the volume; and $\rho^{*}$ is the adiabatically re-sorted density profile, known as the reference density. There are some subtleties when using a nonlinear 230 or compressible equation of state (Saenz et al. 2015). Here, we will only consider a linear and incompressible equation of state.

In an ideal numerical model of the hydrostatic primitive equations that exhibits identically zero mixing, with no buoyancy forcing, every fluid parcel should maintain its temperature and salinity. Under an incompressible equa235 tion of state, the density of any fluid parcel should thus be constant. In this case, 
the adiabatic resorting of the entire fluid yields an unchanging reference density profile and therefore constant RPE, regardless of the actual depth or location of the parcels. Thus, when all parameterised mixing is disabled, and there is no buoyancy forcing, a model with zero spurious mixing will have constant RPE.

Most ocean models experience spurious mixing under nontrivial conditions. By designing experiments with all explicit mixing disabled and without buoyancy forcing, any increase in RPE over time can be attributed to spurious mixing as a result of the model's numerics. For example, a limited advection scheme (which is unable to create water densities outside the existing range) will create some intermediate density class between two pre-existing densities through spurious mixing. The resulting reference density profile will have a higher centre of mass than prior to mixing, which manifests as an increase in RPE. Conversely, RPE can only be decreased by lowering the centre of mass of the adiabatically re-sorted fluid. The disadvantage of the RPE technique is that in order to obtain a meaningful result, explicit mixing and buoyancy forcing must be disabled.

\subsection{The contributions to spurious mixing}

In many models, there is an explicit distinction between the horizontal and vertical dynamics, particularly in models with a generalised vertical coordinate, such as those employing the ALE algorithm. MOM6 performs its regridding/remapping implementation of ALE in a distinct part of a timestep, after the horizontal dynamics have been resolved. Taking advantage of this distinction, we can diagnose the instantaneous RPE at multiple points during a single timestep: at the beginning of a timestep, after horizontal dynamics, and after regridding/remapping has been performed. We denote these three calculations of instantaneous $\mathrm{RPE}$ as $\mathrm{RPE}_{i}, \mathrm{RPE}_{h}$ and $\mathrm{RPE}_{v}$, respectively. The change in RPE due to horizontal dynamics is simply $\mathrm{RPE}_{h}-\mathrm{RPE}_{i}$, and similarly the change due to regridding/remapping is $\mathrm{RPE}_{v}-\mathrm{RPE}_{h}$.

Previous analyses of spurious mixing through changes in RPE have only used a timeseries of RPE taken from a single stage in a timestep. This means that spurious mixing is only diagnosed for the model as a whole, and cannot 


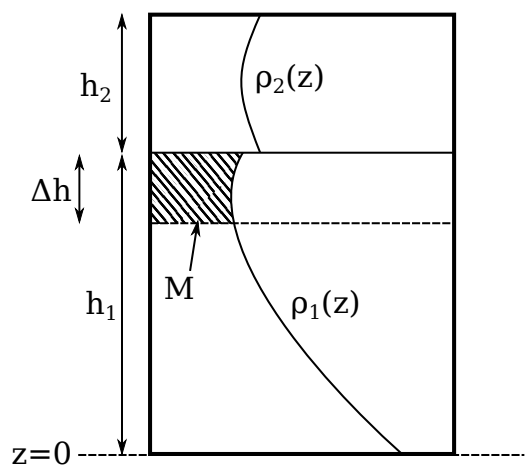

Figure 1: A schematic demonstrating the ability for regridding/remapping to cause a decrease in RPE when using a remapping scheme with a higher reconstruction order than piecewise constant.

be attributed to specific algorithms in the model. Our technique of separating RPE contributions allows us to address the interplay of different components, such as the order of horizontal advection scheme chosen, the order of accuracy of vertical reconstruction used for remapping, and the specific impact of the choice of vertical coordinate. Determining the dominant contributions to spurious mixing is also important because of the anisotropy of horizontal and vertical coordinates in ocean models. Some component of the horizontal spurious mixing may be diapycnal in the presence of sloping isopycnals, and therefore manifests in an RPE change even when a linear equation of state is used. Spurious mixing in the vertical direction, due to regridding/remapping, will directly affect the vertical profile of density and hence RPE.

From a physical viewpoint, we expect mixing processes to produce a monotonic RPE increase with time. However, the vertical process of regridding/remapping can cause RPE to decrease in cases with sub-gridscale representation of tracer that is higher order than piecewise constant. We use a simple example to demonstrate how the combination of regridding/remapping may create a decrease in total potential energy which, for a statically stable single column case, is equivalent to the RPE. 
Figure 1 shows a simple two-cell domain under regridding/remapping. For

After remapping, the potential energy becomes

$$
\begin{aligned}
P E_{f} & =\left(\bar{\rho}_{1} h_{1}-M\right) \frac{h_{1}-\Delta h}{2} \\
& +\left(\bar{\rho}_{2} h_{2}+M\right)\left(h_{1}-\Delta h+\frac{h_{2}+\Delta h}{2}\right) .
\end{aligned}
$$

Taking the difference between the final and initial potential energy gives the change due to regridding/remapping

$$
\begin{aligned}
P E_{f}-P E_{i}= & -\bar{\rho}_{1} h_{1} \frac{\Delta h}{2}-M\left(\frac{h_{1}-\Delta h}{2}\right) \\
& -\bar{\rho}_{2} h_{2} \frac{\Delta h}{2}+M\left(h_{1}-\Delta h+\frac{h_{2}+\Delta h}{2}\right) \\
= & M\left(\frac{h_{1}+h_{2}}{2}\right)-\left(\bar{\rho}_{1} h_{1}+\bar{\rho}_{2} h_{2}\right) \frac{\Delta h}{2} .
\end{aligned}
$$

The potential energy of the domain is calculated using the information carried by the model, namely the cell mean density and cell thickness, and ignores sub-grid reconstruction. Initially, the potential energy in the column in

$$
P E_{i}=\bar{\rho}_{1} h_{1} \frac{h_{1}}{2}+\bar{\rho}_{2} h_{2}\left(h_{1}+\frac{h_{2}}{2}\right) .
$$

PCM is the lowest order reconstruction, and gives $M=\bar{\rho}_{1} \Delta h$, thus $P E_{f}-$ $P E_{i} \geq 0$ with the condition that $\bar{\rho}_{1}>\bar{\rho}_{2}$. However, it is also possible for $P E_{f}-P E_{i}<0$ when the remapping is higher order (e.g. PQM) if the higherorder reconstruction is such that $\frac{M}{\Delta h}<\frac{\bar{\rho}_{1} h_{1}+\bar{\rho}_{2} h_{2}}{h_{1}+h_{2}}$, i.e. if the average density of 


\section{Results}

Experiments are run in MOM6 across three test cases: a lock exchange, internal waves, and baroclinic eddies, chosen to match Ilicak et al. (2012). The model is run using the piecewise parabolic (PPM) horizontal tracer advection scheme, and the PPM $h_{4}$ vertical remapping scheme. Unless otherwise specified, the two vertical coordinates used are z-star with uniform grid spacing, and continuous isopycnal (rho) with linear layer densities. A linear equation of state is used, with constant salinity such that density is a function only of temperature, $\rho=\rho_{0}-\alpha \theta$. Here, $\rho_{0}=1001 \mathrm{~kg} \mathrm{~m}^{-3}$ is the reference density, $\alpha=$ $0.2 \mathrm{~kg} \mathrm{~m}^{-3} \mathrm{~K}^{-1}$ is the thermal expansion coefficient, and $\theta$ is the temperature in degrees Celsius.

All the chosen test cases use flat-bottomed domains, so we present no evaluation of the split RPE method in the presence of topography. The relevant test case from Ilicak et al. (2012) involves convective instabilities, which are often parameterised and lie beyond the scope of this study. However, the split RPE method is extensible to arbitrary domains, using techniques described by Stewart et al. (2014).

\subsection{Lock exchange}

The lock exchange test case (fig. 2) is a simple configuration that shows the 325 creation of intermediate densities by spurious mixing. The test case takes place in a two-dimensional domain of $64 \mathrm{~km}$ width and $20 \mathrm{~m}$ depth. Only the highest resolution experiments investigated by Ilicak et al. (2012) are chosen, with horizontal and vertical grid spacings of $\Delta x=500 \mathrm{~m}$ and $\Delta z=1 \mathrm{~m}$, respectively. 


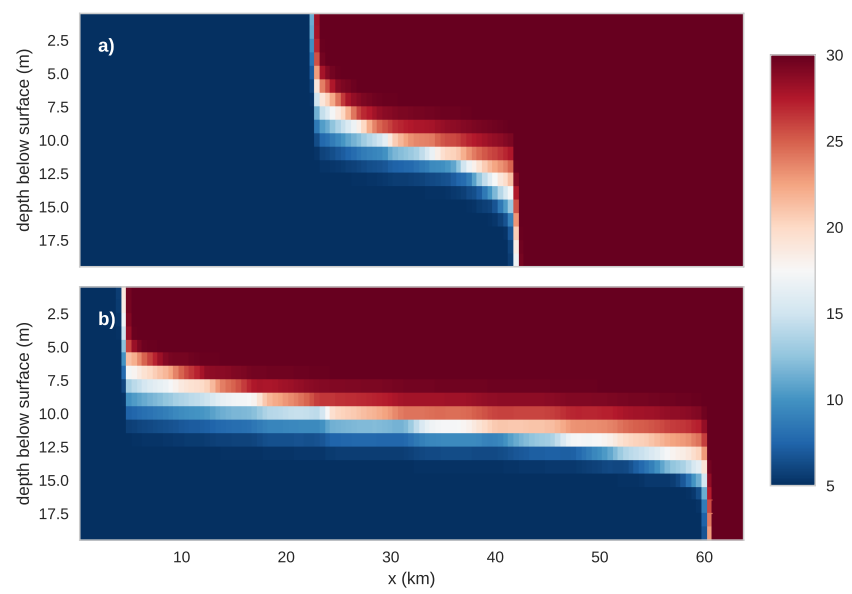

Figure 2: Snapshots of lock exchange in MOM6 at 6 hours (a) and 17 hours (b) at $\nu_{h}=$ $0.01 \mathrm{~m}^{2} \mathrm{~s}^{-1}\left(\operatorname{Re}_{\Delta}=2.5 \times 10^{4}\right)$, using the z-star vertical coordinate. Temperature $\left({ }^{\circ} \mathrm{C}\right)$ is shown in colours.

The lock exchange is defined by an initial temperature distribution comprised of one density class on each side of the domain,

$$
\Theta(x)= \begin{cases}5{ }^{\circ} \mathrm{C} & x<32 \mathrm{~km} \\ 30^{\circ} \mathrm{C} & x \geq 32 \mathrm{~km} .\end{cases}
$$

This case is equivalent to two adjacent basins, each at constant temperature, with a dam between them that is removed at $t=0$. The warm water from the right basin flows from right-to-left above cold water, while conversely cold water from the left basin flows underneath the warm water from left-to-right (fig. 2). The flow is a gravity current, for which we have a theoretical prediction for the front velocity in a rectangular channel, given by

$$
u_{f}=\frac{1}{2} \sqrt{g H \Delta \rho / \rho_{0}},
$$

where $H$ is the domain depth and $\Delta \rho$ is the density difference across the front, normalised by a reference density, $\rho_{0}$ (Ilicak et al. 2012). However, the expected solution of this test case differs from a physical gravity current in that we expect there to be no region of turbulent mixing, and therefore no intermediate 
densities, behind the head of the current, as we have disabled all explicit and parameterised mixing.

When calculating the grid Reynolds number, the theoretical front velocity is used as the characteristic velocity scale, to match Ilicak et al. (2012). All runs were carried out for 17 hours using a baroclinic timestep that satisfied CFL conditions across the range of horizontal viscosities $(0.01,0.1,1,10,100$ and $\left.200 \mathrm{~m}^{2} \mathrm{~s}^{-1}\right)$.

The time series of normalised RPE (normalised by initial condition RPE) in fig. 3a shows MOM6 having a similar shape to MITGCM and MOM5. The actual values of the normalised RPE are much lower in MOM6, showing that less spurious mixing occurs in MOM6. Figure $3 \mathrm{~b}$ shows the dependence on grid Reynolds number $\operatorname{Re}_{\Delta}$ of the rate of change of RPE at 17h. MOM6 performs better than the other models over the range $\operatorname{Re}_{\Delta}>2$. To better understand why MOM6 performs so well in this case, we next look at the sensitivity to advection order and the horizontal/vertical contributions to spurious mixing.

\subsubsection{Advection order}

One aspect of model configuration that may affect spurious mixing is the order of accuracy of the tracer advection scheme. A higher-order advection scheme purports to reduce the spurious mixing in advection, at the cost of runtime performance. Two of the advection schemes in MOM6 have been tested here, the quasi-fourth-order limited piecewise parabolic method (PPM) (Colella and Woodward 1984) and the Huynh third-order piecewise parabolic scheme, PPM:H3 (Huynh 1997). They exhibit nearly identical spurious mixing for all tested values of $\operatorname{Re}_{\Delta}$ in the lock exchange (fig. $3 \mathrm{~b}$ ). This result suggests that both schemes are operating in the flux-limited regime near the temperature front, where the order of reconstruction has a reduced impact on spurious mixing. Note that the rate of change of RPE used in this test case is the instantaneous rate during the last timestep, so although PPM:H3 appears more accurate, the total increase in RPE is greater (not shown). 

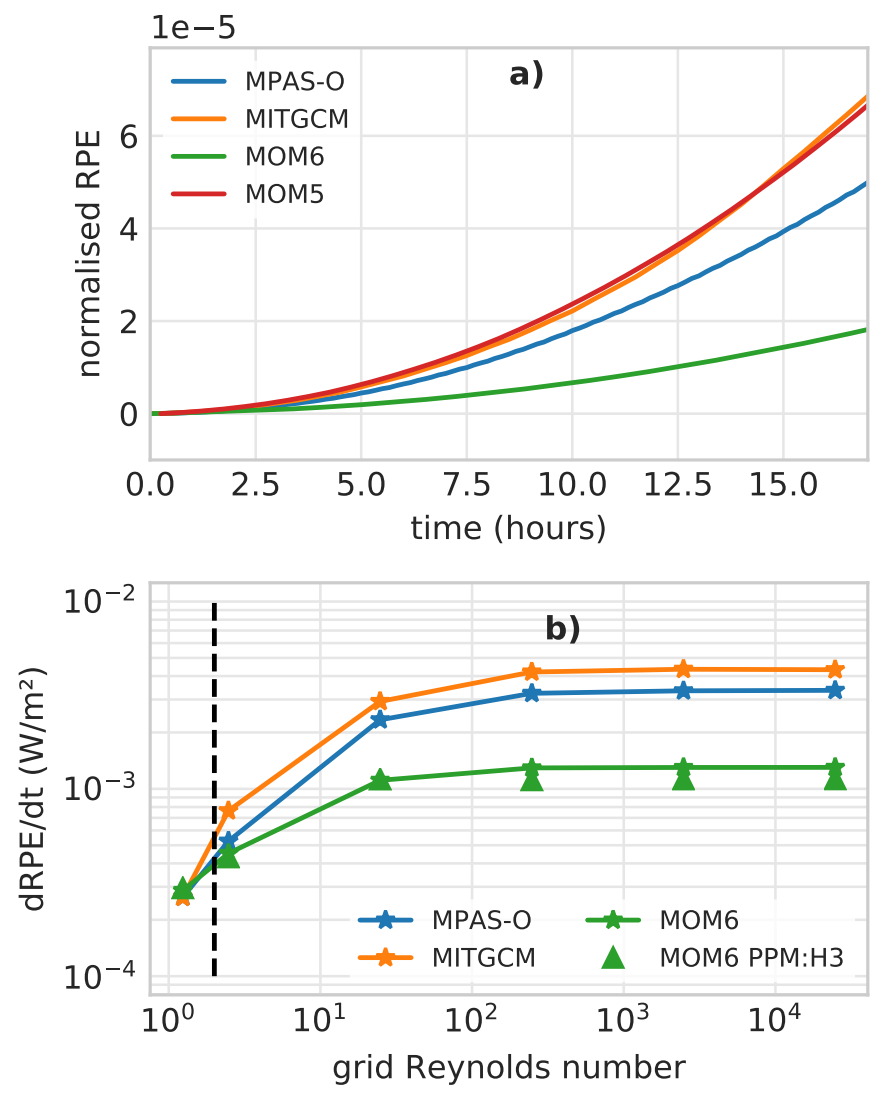

Figure 3: (a) Normalised RPE evolution for $\nu_{h}=0.01 \mathrm{~m}^{2} \mathrm{~s}^{-1}\left(\operatorname{Re}_{\Delta}=2.5 \times 10^{4}\right)$ in the lock exchange. MITGCM and MOM5 results come from Ilicak et al. (2012), while MPAS-O results come from Petersen et al. (2015). (b) Instantaneous rate of RPE change at $17 \mathrm{~h}$ in the lock exchange for MPAS-O, MITGCM and MOM6 using the z-star vertical coordinate. The dashed line is at $\operatorname{Re}_{\Delta}=2$ showing the necessary condition for viscosity to damp grid-scale noise. 


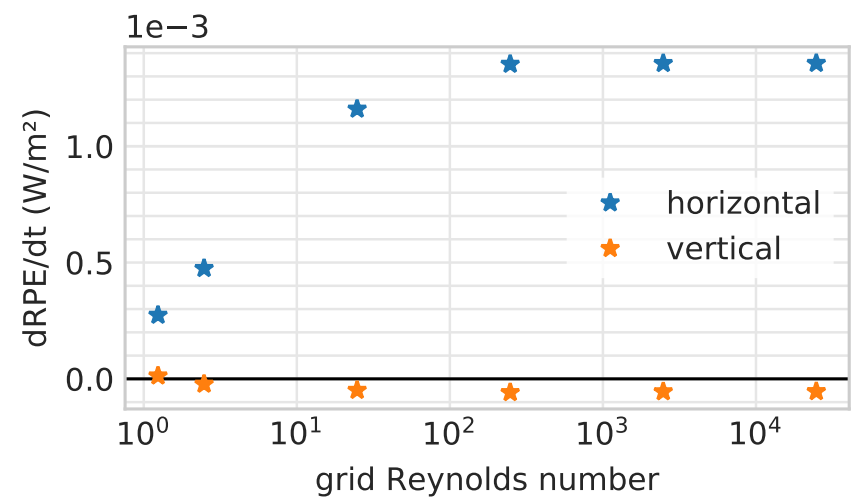

Figure 4: Horizontal and vertical contributions to the instantaneous rate of RPE change in MOM6 in the lock exchange at $17 \mathrm{~h}$.

\subsubsection{Spurious mixing contributions}

Figure 4 shows that the mixing is predominantly due to horizontal processes. This appears to be at odds with the conclusions of Ilicak et al. (2012), who found that "high spatial variability in the vertical velocities between the fronts is therefore the dominant cause of spurious diapycnal mixing in this test case." However, MOM6 uses an upstream momentum advection scheme that reduces spatial variability in the velocity field between the fronts. Vertical velocities in a layered model manifest as changes in local layer thickness, which are redistributed in the regridding/remapping step. However, the spurious mixing across the fronts due to horizontal tracer advection is the leading-order effect. Indeed, for all of the experiments, the average RPE change due to regridding/remapping is negative. Physically, this means that regridding/remapping tends to slightly lower the centre of mass of the domain, counteracting some of the centre of mass increase due to mixing by the advection scheme. The magnitude of this compensation by regridding/remapping is negligible, so the majority of the spurious mixing still occurs during horizontal tracer advection. 


\subsection{Internal waves}

The breaking of nonlinear internal waves in the ocean is a significant source of abyssal mixing, and thus is an important process contributing to the abyssal ocean circulation (Waterhouse et al. 2014). While ocean models do not, in general, represent the breaking of nonlinear internal waves, the propagation of linear internal waves can produce spurious vertical mixing in ocean models, particularly with vertical grids that do not directly represent their propagation, such as z-star (Gouillon 2010), which is fixed with respect to non-barotropic motion. However, other vertical coordinates, such as z-tilde, permit layers to move with the waves, thereby restricting transport between layers and ultimately reducing spurious mixing.

This test case is configured as presented by Ilicak et al. (2012). It consists of a linearly stratified background temperature distribution in a domain $500 \mathrm{~m}$ deep and $250 \mathrm{~km}$ wide (fig. 5a). The horizontal grid spacing is $5 \mathrm{~km}$, and the vertical grid spacing $\Delta z$ is $25 \mathrm{~m}$. A wave perturbation is superimposed, lifting the isopycnals in the centre of the domain to set up counter-propagating internal waves towards the left and right horizontal boundaries. This initial condition can be achieved in two separate ways: by altering the thicknesses of constant temperature layers (layered IC), and by altering the temperature in constant thickness layers (level IC).

Experiments were run with horizontal viscosities $\nu_{h}$ of $0.01,1,15$ and $150 \mathrm{~m}^{2} \mathrm{~s}^{-1}$, for z-star, z-tilde (using level and layered initial conditions), and continuous isopycnal coordinates. The test case was run for 100 days to allow the waves to propagate many times across the full extent of the domain. The first 10 days are treated as spin-up, however, so the characteristic velocity scale was calculated from the mean domain-averaged kinetic energy from 10 to 100 d. Similarly, the rate of change of RPE was averaged over this same period.

Comparing the model state after the full $100 \mathrm{~d}$ evolution (figs. 5b to $5 \mathrm{~d}$ ) reveals the impact of the choice of vertical coordinate. Coordinate surfaces are misaligned with temperature surfaces in z-star, thus there is lateral advection and thus diffusion associated with the gradient within layers. The z-tilde and 

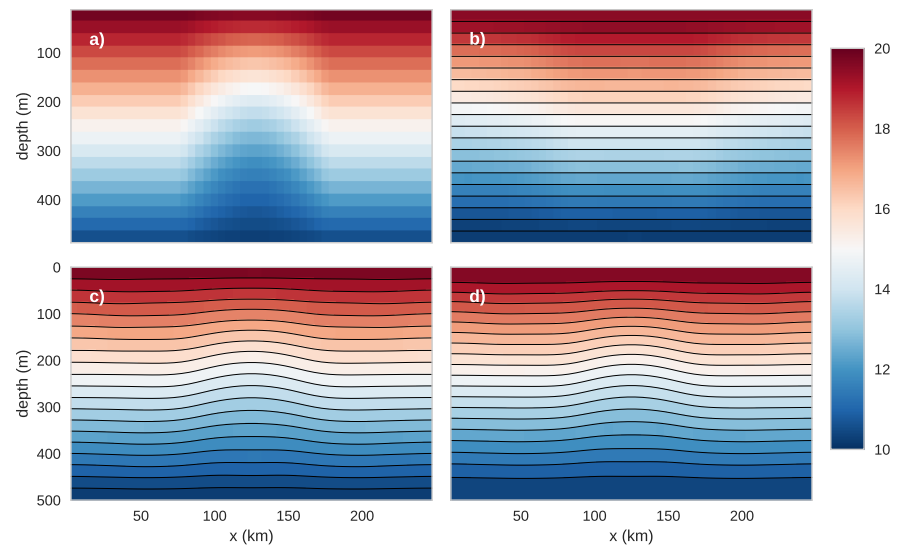

Figure 5: Snapshot of the internal wave level initial condition (a), and state in MOM6 after 100 days for different vertical coordinates: z-star (b), z-tilde (layered initial condition) (c) and continuous isopycnal (d). Temperature $\left({ }^{\circ} \mathrm{C}\right)$ is shown in colours, and interfaces are shown with black lines.

continuous isopycnal coordinates further reduce along-layer temperature gradients and therefore lead to reduced spurious mixing and better preserve the vertical stratification.

It is important to note here that the solution varies substantially depending on the choice of coordinate (figs. $5 \mathrm{~b}$ to $5 \mathrm{~d}$ ). Due to the dispersive nature of the internal wave problem, we can't expect the solution to remain fully monochromatic when integrated by numerical methods. Using an isopycnal coordinate would give a solution accurate to second order. Additionally, when the z-tilde simulation is initialised from a level initial condition (not pictured), temperature within layers is non-uniform in the final solution.

With regard to the total rate of spurious mixing, MOM6 performs better than all the other tested models across the full range of horizontal viscosities with all three of the z-star, z-tilde and continuous isopycnal vertical coordinates (fig. 6a). As a quasi-Lagrangian model, vertical layers in MOM6 are able to move within their column as waves pass through during the dynamics phase of a single timestep, regardless of the chosen coordinate. As this is an adiabatic process, there is no spurious mixing from vertical motions before horizontal tracer 


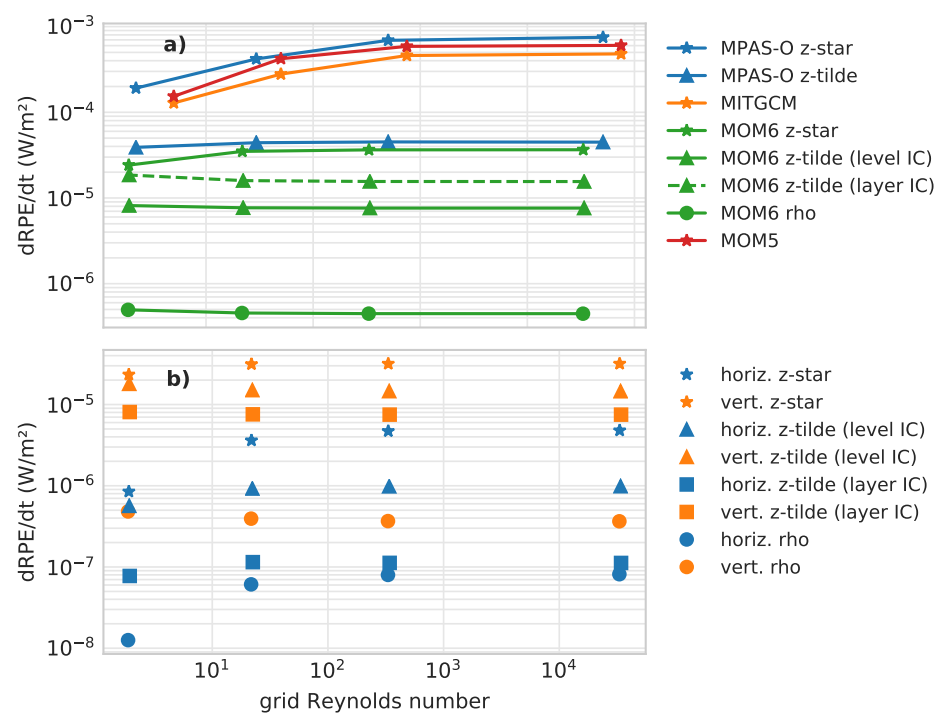

Figure 6: (a) Averaged rate of RPE change from 10 to 100 days in the internal waves test case. MITGCM and MOM5 results come from Ilicak et al. (2012), while MPAS-O results come from Petersen et al. (2015). MPAS-O z-tilde uses $\tau_{D l f}=100 \mathrm{~d}$. (b) Contributions to spurious mixing by horizontal and vertical processes in the internal waves test case in MOM6. Each contribution is the time-averaged total rate of RPE change from 10 to 100 days.

advection is performed. The temperature structure that existed at the beginning of the dynamics phase is retained, thus the local lateral gradients along layers are weaker than they would be, had vertical advection been performed. As a consequence, the spurious mixing due to horizontal tracer advection itself is reduced. The more dynamically-relevant temperature gradient in this test case exists in the vertical direction, and we can conclude from this result that the Lagrangian tendency of ALE permits lower spurious mixing in the presence of vertical motions. Indeed, we will see that the contribution from horizontal tracer advection becomes significantly smaller than that of remapping. This inference is further supported by the successive reductions in spurious mixing with the z-tilde and continuous isopycnal (rho) vertical coordinates, which are more Lagrangian and isopycnal, respectively, than z-star.

As MOM6 uses a quasi-Lagrangian ALE implementation, vertical velocities 
are implicit in the regridding/remapping stage. Additionally, motions are Lagrangian within a single timestep. The simplified z-tilde coordinate in the model can then be described as

$$
\tilde{z}_{k}^{n+1}=\tilde{z}_{k}^{n}+\frac{\Delta t}{\tau_{h h f}}\left(z_{k}^{*}-\tilde{z}_{k}^{n}\right),
$$

450 a modest improvement in the vertical contribution. As this test case is dominated by the vertical spurious mixing, it is only this modest improvement that shows up in the total spurious mixing. Initialising this test case from a layered 
a more significant impact on spurious mixing.

As the internal waves test case involves primarily vertical motions, the alonglayer gradients remain small regardless of the chosen vertical coordinate. The spurious mixing due to horizontal tracer advection is approximately an order of magnitude lower than that due to regridding/remapping. The z-tilde and continuous isopycnal coordinates are strongly Lagrangian in this test case, which, without any explicit mixing leads to very little along-layer advection of temperature (equivalent to density in this test case). As expected, the spurious mixing due to horizontal tracer advection with the z-star coordinate is much higher, up to 5 times that of the more Lagrangian coordinates.

\subsection{Baroclinic eddies}

The previous two test cases were two-dimensional and also did not incorporate the influence of the Coriolis force. We now introduce a third test case from Ilicak et al. (2012) that involves a baroclinically unstable temperature front in 490 a periodic channel with rotation, described by Petersen et al. (2015). This front generates vigorous eddying without either mechanical or buoyancy forcing, thus it is a closed system suitable for analysis by changes in RPE. The domain is a periodic channel in the $x$ direction, $160 \mathrm{~km}$ wide by $500 \mathrm{~km}$ long, with a depth of $1000 \mathrm{~m}$ at vertical resolution $\Delta z=50 \mathrm{~m}$ (fig. 7a).

In order to encourage baroclinicity, a strong quadratic bottom drag with drag coefficient $C_{D}=0.01$ is used. Experiments were performed at horizontal resolutions of 1, 4 and $10 \mathrm{~km}$. For each choice of horizontal resolution, the horizontal viscosities $\nu_{h}$ were $1,5,10,20$ and $200 \mathrm{~m}^{2} \mathrm{~s}^{-1}$, giving a range of lateral grid Reynolds numbers from $O(1)$ at the highest viscosity, through to $O(1000)$

istic velocity scale was calculated from the mean of the domain-averaged kinetic energy.

Figures $7 \mathrm{~b}$ and $7 \mathrm{c}$ show the surface temperature after the full 320 days of simulation at $1 \mathrm{~km}$ horizontal resolution, at the lowest and highest viscosities, 


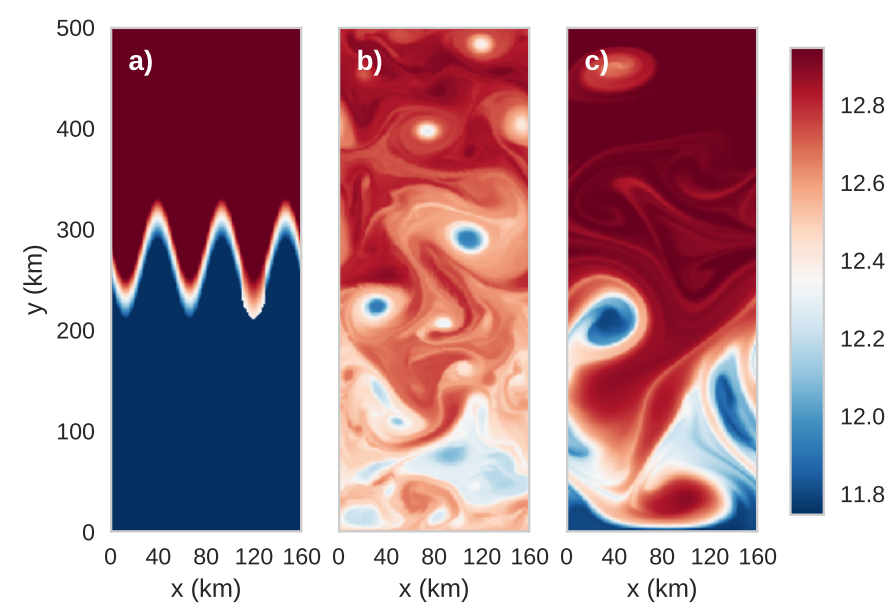

Figure 7: (a) Snapshot of initial condition of surface temperature for the baroclinic eddies test case in MOM6 at $1 \mathrm{~km}$ horizontal resolution. The temperature perturbation can be seen at the third trough in the sinusoidal front. Snapshots of surface temperature $\left({ }^{\circ} \mathrm{C}\right)$ after 320 days of simulation at $1 \mathrm{~km}$ horizontal resolution, for (b) $\nu_{h}=1 \mathrm{~m}^{2} \mathrm{~s}^{-1}\left(\operatorname{Re}_{\Delta}=88\right)$ and (c) $\nu_{h}=200 \mathrm{~m}^{2} \mathrm{~s}^{-1}\left(\operatorname{Re}_{\Delta}=0.25\right)$.

${ }_{505} 1 \mathrm{~m}^{2} \mathrm{~s}^{-1}$ and $200 \mathrm{~m}^{2} \mathrm{~s}^{-1}$, respectively. In the low viscosity case, stronger spurious mixing has occurred, but finer-scale features are also evident. Conversely, the volume of mixed fluid is significantly less with a higher horizontal viscosity, but the eddies are much weaker due to the momentum damping by the viscosity. Due to the eddying nature of this simulation, the solution is strongly affected by the momentum closure, which is set by the viscosity. Identifying any changes in the solution due to spurious mixing itself is a secondary concern in this study.

At all horizontal resolutions, the spurious mixing in MOM6 (z-star), MOM5 and MITGCM is very similar (fig. 8), with a modest improvement in MOM6 over MITGCM in many cases. However, the differences between these models are marginal compared to MPAS-O, as discussed by Petersen et al. (2015). For all cases in MOM6 with the continuous isopycnal coordinate, the total average rate of RPE change is negative, so it is not shown in fig. 8. In this configuration, the magnitude of the RPE change varies from half to an order of magnitude lower than the z-star configuration. As the differences in the models except 

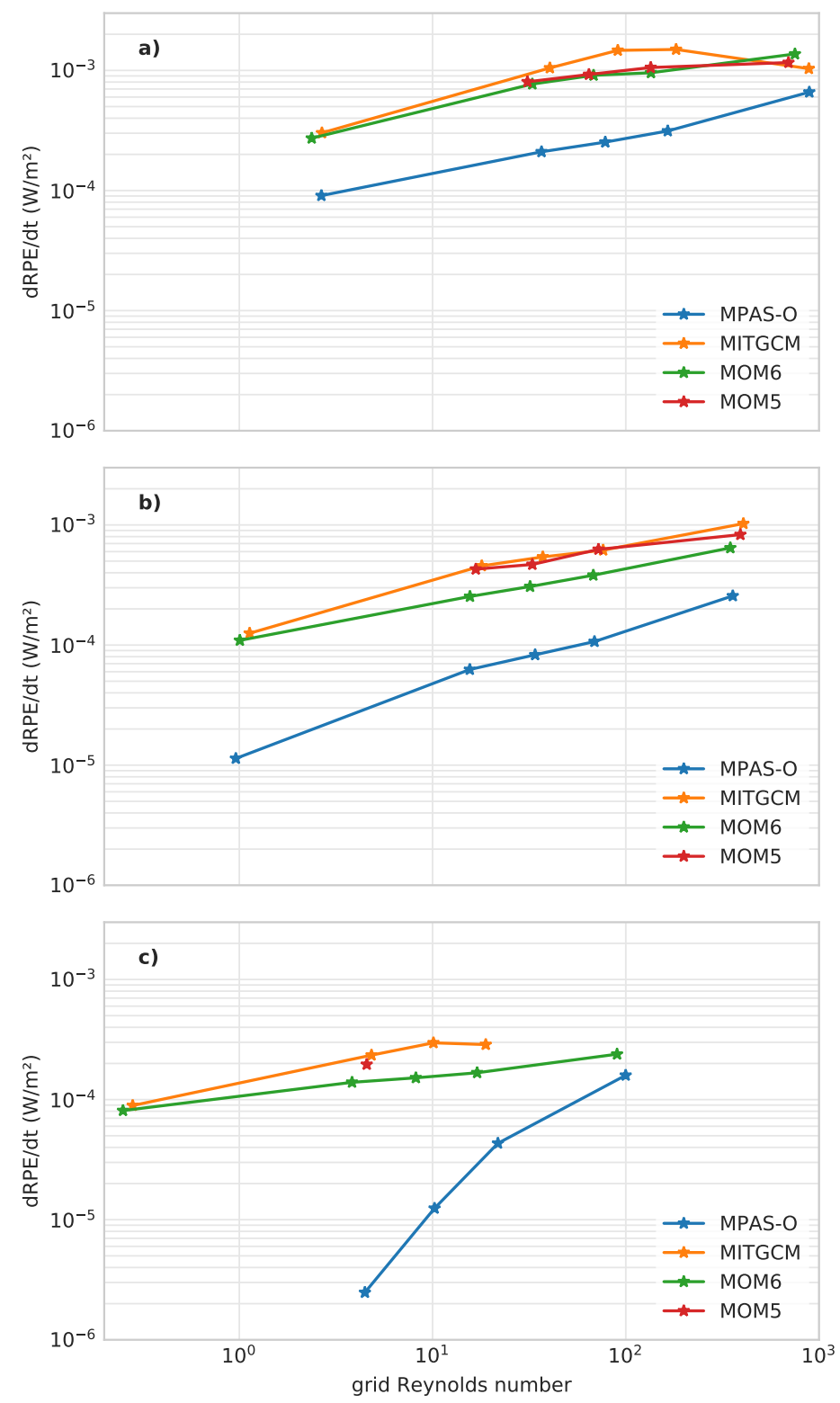

Figure 8: Average rate of RPE change for all baroclinic eddy experiments over the entire 320 day run. MITGCM and MOM5 results come from Ilicak et al. (2012), while MPAS-O results are from Petersen et al. (2015). (a) $\Delta x=10 \mathrm{~km}$, (b) $\Delta x=4 \mathrm{~km}$, (c) $\Delta x=1 \mathrm{~km}$. 

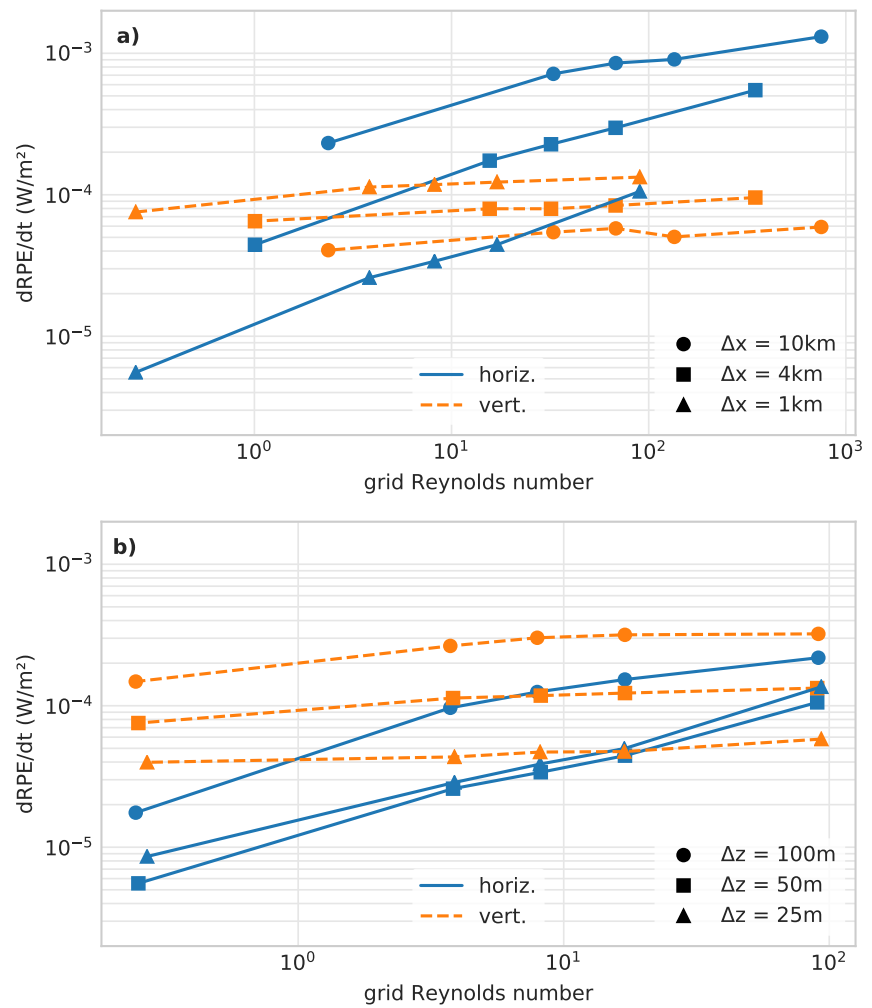

Figure 9: Average rates of RPE change for horizontal and vertical processes over full simulation of the baroclinic eddies test case, showing spurious mixing contributions in MOM6 for (a) each horizontal resolution at $50 \mathrm{~m}$ vertical resolution across the range of horizontal viscosities, and (b) varying vertical resolution at $1 \mathrm{~km}$ horizontal resolution.

MPAS-O is slight, we instead turn our attention to the contributions to the spurious mixing in MOM6 and the impact of resolution.

\subsubsection{Spurious mixing contributions}

We consider the contributions to spurious mixing at each of the tested horizontal resolutions $(1,4$ and $10 \mathrm{~km})$ in fig. 9a. As horizontal resolution increases 525 (grid spacing decreases), the spurious mixing present in horizontal tracer advection decreases, accompanied by a smaller increase in the spurious mixing by regridding/remapping. Therefore the fraction of the total spurious mixing con- 
tributed by regridding/remapping increases with horizontal resolution. Indeed, at $1 \mathrm{~km}$ horizontal resolution, regridding/remapping contributes to the majority of the total spurious mixing across the full range of viscosities. The fact that the spurious mixing contributed by regridding/remapping stays relatively constant suggests that the implicit vertical velocities are physical, occurring with larger horizontal scales than the grid-scale. However, we speculate that the increase of this contribution as horizontal resolution decreases can be attributed in part due to the larger number of water columns in which regridding/remapping must be applied. If there is a per-column baseline spurious mixing due to remapping, this would show up as a nonlinear contribution which is affected by resolution.

As shown in fig. 9a, the vertical component of spurious mixing only approximately doubles for a tenfold increase in horizontal resolution. Figure $9 \mathrm{~b}$ shows the effect of doubling $(\Delta z=25 \mathrm{~m})$ and halving $(\Delta z=100 \mathrm{~m})$ the vertical resolution, at a constant $1 \mathrm{~km}$ horizontal resolution. With insufficient vertical resolution to fully resolve the baroclinic structure of the flow $(\Delta z=100 \mathrm{~m})$, spurious mixing due to horizontal tracer advection is elevated, however the convergence of the horizontal contributions at $\Delta z=25$ and $50 \mathrm{~m}$ shows that excess vertical resolution has little impact. However, the vertical contribution continues to improve as resolution increases. This benefit is fairly isolated, as the flow is already resolved in both the lateral and vertical grids. This result demonstrates that increasing vertical resolution provides another way of reducing spurious mixing for a given vertical coordinate, which is particularly important at high horizontal resolutions, such as the transition to eddy resolving in the horizontal grid, shown here.

\section{Discussion}

Due to the aspect ratio of the ocean, horizontal and vertical dynamics are implemented separately, and often with different algorithms in ocean models. However, spurious mixing has not been attributed to specific processes such as horizontal advection or vertical regridding/remapping. The technique we have 
developed here determines the contributions to changes of RPE which allows for diagnosis and attribution of spurious mixing within a model. We applied this technique to determine the contributions to spurious mixing present in MOM6 in a suite of three test cases (lock exchange, internal waves, and baroclinic eddies). The results raise some points of discussion regarding horizontal and vertical processes, and how they interact.

High-order horizontal tracer advection schemes are often employed to reduce the horizontal component of spurious mixing. However, the two tracer advection schemes currently present in MOM6 (PPM and PPM:H3) exhibit very similar magnitudes of spurious mixing. Consistent with the findings of Ilicak et al. (2012), reduction of grid-scale variance in the velocity field is a more important effect.

With increasing horizontal resolution, the rate of horizontal spurious mixing decreases (fig. 8). However, the rate of vertical spurious mixing is relatively insensitive to horizontal resolution. Therefore, as horizontal resolution increases, so does the fraction of spurious mixing contributed by vertical processes. As models tend toward higher horizontal resolution, the spurious mixing baseline can be improved through configuration choices and the algorithms relating to the vertical processes. These include the order of accuracy of remapping, and choice of vertical resolution, which has a direct impact on the rate of vertical spurious mixing. Further development may be focused on vertical coordinates that permit an accurate resolution of the underlying flow, without introducing excess spurious mixing.

Another important contribution to the magnitude of spurious mixing is the choice of vertical coordinate. Using the internal gravity waves test case, it was shown that generalised coordinates such as z-tilde or continuous isopycnal yield some improvements in spurious mixing over the ubiquitous z-star coordinate used in ocean models (fig. 6). When using the z-tilde vertical coordinate, there are reductions in both the vertical and horizontal components of spurious mixing. The reduction in the horizontal component comes from the homogeneity of temperature within layers, as coordinate surfaces are more aligned with den- 
sity surfaces. When layers become nearly-isopycnal, errors in the lateral tracer advection scheme are reduced. This error reduction is more apparent with the continuous isopycnal coordinate. Additionally, the vertical component of spurious mixing with z-tilde is lower than that of z-star, although the magnitude of this effect is significantly smaller than the horizontal. Due to the restoring timescale of the grid, the grid moves less during regridding than z-star (which is equivalent to z-tilde with an instant restoring timescale, i.e. $\tau_{h h f}<\Delta t$, where $\Delta t$ is the baroclinic model timestep). In turn, the reconstruction errors accumulated during remapping are smaller. In the limit of infinite restoring timescale (a fully-Lagrangian grid), there is no movement during regridding, hence remapping is a null operation and there is zero vertical spurious mixing.

Calculating the contributions to spurious mixing using a part-timestep RPE analysis is a new technique that allows for more refined attribution of the impact of model configuration on both horizontal and vertical spurious mixing. In particular, the choice of horizontal and vertical resolution, horizontal tracer advection scheme and vertical coordinate all have different effects on the two components of spurious mixing. As models tend toward higher horizontal resolution, the vertical resolution and the vertical coordinate become increasingly important configuration choices, and should be a key consideration for those developing models.

\section{Acknowledgements}

We thank the three anonymous reviewers and the editor for their valuable feedback. This study was supported by the Australian Research Council Centre of Excellence for Climate System Science grant CE110001028, and an Australian Government Research Training Program (RTP) Scholarship. The computational modelling was supported by the NCI National Facility at ANU, Australia. 


\section{References}

Adcroft, A., Campin, J.-M., 2004. Rescaled height coordinates for accurate representation of free-surface flows in ocean circulation models. Ocean Modelling 7 (3-4), 269-284.

Armour, K. C., Marshall, J., Scott, J. R., Donohoe, A., Newsom, E. R., 2016. Southern Ocean warming delayed by circumpolar upwelling and equatorward transport. Nature Geoscience 9 (7), 549-554.

Bleck, R., 2002. An oceanic general circulation model framed in hybrid isopycnic-Cartesian coordinates. Ocean Modelling 4 (1), 55-88.

Burchard, H., Rennau, H., 2008. Comparative quantification of physically and numerically induced mixing in ocean models. Ocean Modelling 20 (3), 293311.

Colella, P., Woodward, P. R., 1984. The Piecewise Parabolic Method (PPM) for gas-dynamical simulations. Journal of Computational Physics 54 (1), 174201.

Daru, V., Tenaud, C., 2004. High order one-step monotonicity-preserving schemes for unsteady compressible flow calculations. Journal of Computational Physics 193 (2), 563-594.

Gent, P. R., McWilliams, J. C., 1990. Isopycnal Mixing in Ocean Circulation Models. Journal of Physical Oceanography 20 (1), 150-155.

Gentry, R. A., Martin, R. E., Daly, B. J., 1966. An Eulerian differencing method for unsteady compressible flow problems. Journal of Computational Physics $1(1), 87-118$.

Gouillon, F., 2010. Internal Wave Propagation and Numerically Induced Diapycnal Mixing in Oceanic General Circulation Models. Ph.D. thesis, Florida State University. 
Griffies, S. M., 2004. Fundamentals of Ocean Climate Models. Princeton University Press, Princeton, N.J, oCLC: ocm54487392.

Griffies, S. M., Pacanowski, R. C., Hallberg, R. W., 2000. Spurious Diapycnal Mixing Associated with Advection in a z-Coordinate Ocean Model. Monthly Weather Review 128 (3), 538-564.

Griffies, S. M., Winton, M., Anderson, W. G., Benson, R., Delworth, T. L., Dufour, C. O., Dunne, J. P., Goddard, P., Morrison, A. K., Rosati, A., Wittenberg, A. T., Yin, J., Zhang, R., 2015. Impacts on Ocean Heat from Transient Mesoscale Eddies in a Hierarchy of Climate Models. Journal of Climate 28 (3), 952-977.

Hirt, C. W., Amsden, A. A., Cook, J. L., 1974. An arbitrary LagrangianEulerian computing method for all flow speeds. Journal of Computational Physics 14 (3), 227-253.

Huynh, H. T., 1997. Schemes and constraints for advection. In: Fifteenth International Conference on Numerical Methods in Fluid Dynamics. Springer Berlin Heidelberg, pp. 498-503.

Ilıcak, M., 2016. Quantifying spatial distribution of spurious mixing in ocean models. Ocean Modelling 108, 30-38.

Ilıcak, M., Adcroft, A. J., Griffies, S. M., Hallberg, R. W., 2012. Spurious dianeutral mixing and the role of momentum closure. Ocean Modelling 45$46,37-58$.

Jansen, M. F., Adcroft, A. J., Hallberg, R., Held, I. M., 2015. Parameterization of eddy fluxes based on a mesoscale energy budget. Ocean Modelling 92, 2841.

Kasahara, A., 1974. Various Vertical Coordinate Systems Used for Numerical Weather Prediction. Monthly Weather Review 102 (7), 509-522. 
Klingbeil, K., Mohammadi-Aragh, M., Gräwe, U., Burchard, H., 2014. Quantification of spurious dissipation and mixing - Discrete variance decay in a Finite-Volume framework. Ocean Modelling 81, 49-64.

Leclair, M., Madec, G., 2011. Z-Coordinate, an Arbitrary Lagrangian-Eulerian coordinate separating high and low frequency motions. Ocean Modelling $37(3-4), 139-152$.

Margolin, L. G., Shashkov, M., 2003. Second-order sign-preserving conservative interpolation (remapping) on general grids. Journal of Computational Physics 184 (1), 266-298.

Mashayek, A., Ferrari, R., Nikurashin, M., Peltier, W. R., 2015. Influence of Enhanced Abyssal Diapycnal Mixing on Stratification and the Ocean Overturning Circulation. Journal of Physical Oceanography 45 (10), 2580-2597.

Morales Maqueda, M. A., Holloway, G., 2006. Second-order moment advection scheme applied to Arctic Ocean simulation. Ocean Modelling 14 (3-4), 197221.

Petersen, M. R., Jacobsen, D. W., Ringler, T. D., Hecht, M. W., Maltrud, M. E., 2015. Evaluation of the arbitrary Lagrangian-Eulerian vertical coordinate method in the MPAS-Ocean model. Ocean Modelling 86, 93-113.

Prather, M. J., 1986. Numerical advection by conservation of second-order moments. Journal of Geophysical Research: Atmospheres 91 (D6), 6671-6681.

Ringler, T., Petersen, M., Higdon, R. L., Jacobsen, D., Jones, P. W., Maltrud, M., 2013. A multi-resolution approach to global ocean modeling. Ocean Modelling 69, 211-232.

Saenz, J. A., Tailleux, R., Butler, E. D., Hughes, G. O., Oliver, K. I. C., 2015. Estimating Lorenz's Reference State in an Ocean with a Nonlinear Equation of State for Seawater. Journal of Physical Oceanography 45 (5), 1242-1257. 
Stewart, K. D., Saenz, J. A., Hogg, A. M., Hughes, G. O., Griffiths, R. W., 2014. Effect of topographic barriers on the rates of available potential energy conversion of the oceans. Ocean Modelling 76, 31-42.

Vreugdenhil, C. A., Hogg, A. M., Griffiths, R. W., Hughes, G. O., 2015. Adjustment of the Meridional Overturning Circulation and Its Dependence on Depth of Mixing. Journal of Physical Oceanography 46 (3), 731-747.

Waterhouse, A. F., MacKinnon, J. A., Nash, J. D., Alford, M. H., Kunze, E., Simmons, H. L., Polzin, K. L., St. Laurent, L. C., Sun, O. M., Pinkel, R., Talley, L. D., Whalen, C. B., Huussen, T. N., Carter, G. S., Fer, I., Waterman, S., Naveira Garabato, A. C., Sanford, T. B., Lee, C. M., 2014. Global Patterns of Diapycnal Mixing from Measurements of the Turbulent Dissipation Rate. Journal of Physical Oceanography 44 (7), 1854-1872.

White, L., Adcroft, A., 2008. A high-order finite volume remapping scheme for nonuniform grids: The piecewise quartic method (PQM). Journal of Computational Physics 227 (15), 7394-7422.

White, L., Adcroft, A., Hallberg, R., 2009. High-order regridding-remapping schemes for continuous isopycnal and generalized coordinates in ocean models. Journal of Computational Physics 228 (23), 8665-8692.

Winters, K. B., Lombard, P. N., Riley, J. J., D'Asaro, E. A., 1995. Available potential energy and mixing in density-stratified fluids. Journal of Fluid Mechanics $289,115-128$. 